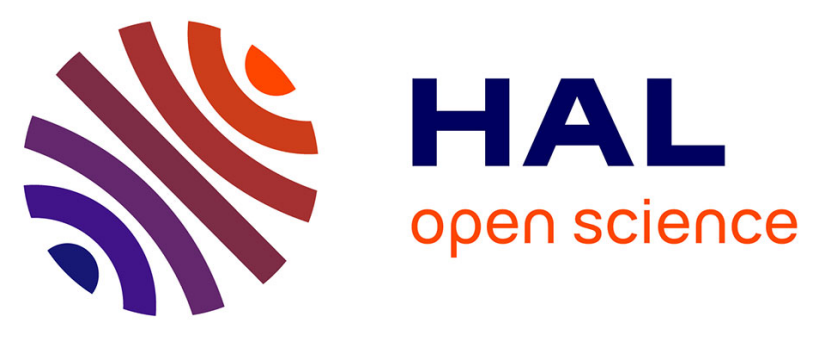

\title{
Direction-Aided Indoor Positioning Leveraging Ultra-Wideband Radio
}

\author{
Mamadou Lamarana Diallo, Antoine Courtay, Mickael Le Gentil, Olivier
} Berder

\section{- To cite this version:}

Mamadou Lamarana Diallo, Antoine Courtay, Mickael Le Gentil, Olivier Berder. Direction-Aided Indoor Positioning Leveraging Ultra-Wideband Radio. 2019 IEEE 90th Vehicular Technology Conference (VTC2019-Fall), Sep 2019, Honolulu, United States. pp.1-6, 10.1109/VTCFall.2019.8891116 . hal-02367861

\section{HAL Id: hal-02367861 \\ https://hal.science/hal-02367861}

Submitted on 18 Nov 2019

HAL is a multi-disciplinary open access archive for the deposit and dissemination of scientific research documents, whether they are published or not. The documents may come from teaching and research institutions in France or abroad, or from public or private research centers.
L'archive ouverte pluridisciplinaire HAL, est destinée au dépôt et à la diffusion de documents scientifiques de niveau recherche, publiés ou non, émanant des établissements d'enseignement et de recherche français ou étrangers, des laboratoires publics ou privés. 


\title{
Direction-Aided Indoor Positioning Leveraging Ultra-Wideband Radio
}

\author{
Mamadou Lamarana Diallo, Antoine Courtay, Mickaël Le Gentil and Olivier Berder \\ Univ Rennes, CNRS, IRISA \\ Email: mamadou.diallo@irisa.fr, antoine.courtay@irisa.fr, mickael.le-gentil@irisa.fr, olivier.berder@irisa.fr
}

\begin{abstract}
Many emerging applications based on robotics or augmented reality (e.g. for museums, BIM...) require accurate indoor positioning, that obviously can not be achieved by global positioning systems or traditional radio systems using received signal strength indicators. Ultra-wideband radio is a promising technology to enhance this accuracy, but its robustness still suffers in noisy environment and most of localization algorithms keep a prohibitive complexity to be embedded on target nodes. The Newton-Gauss algorithm represents a good trade-off between positioning performance and processing needs at the node level. As some recent wireless body area networks embed inertial measurement unit, the target direction can be used to further enhance the accuracy while keeping an acceptable complexity. In fact, the direction allows to narrow the research area of the mobile position. Thanks to this additional information, our direction-aided Newton-Gauss algorithm allows a gain of more than $14 \%$ in terms of accuracy over classical Newton-Gauss algorithm.
\end{abstract}

\section{INTRODUCTION}

Location-based service is one of the newly emerging technologies having potential for numerous applications in the commercial and public safety fields. For example, museum needs a visitor tracking (e.g. his position and the artistic work he is watching for) to achieve a virtual and/or augmented experience. In indoor context, the traditional global positioning system is not suitable for various reasons such as cost, accuracy, and mostly its unavailability. Thus, Local Positioning System (LPS) must use others technologies like video tracking or radio-electrical waves. As we target accurate low power system and low complexity algorithms, UltraWideband (UWB) radio is a good candidate [1].

To boost the LPS accuracy for such applications, several strategies can be considered: (a) a specific setting of anchors in the room, and (b) the measurement that will be traduced into distance, e.g. Time Of Arrival (TOA) or Time Of Flight (TOF), Time Difference Of Arrival (TDOA), Angle Of Arrival (AOA) [2], and (c) the choice of the most adapted algorithm to obtain the target position from several distance estimations. For the latter, there are numerous solutions in the literature. The reader may refer to [3], wherein a classification of the different methods or algorithms for indoor positioning has been carried out. Some of these methods are adapted to the deployed LPS. For instance, trilateration is used when the measurements of the receive signal strength indicator [4], the TOA or the TDOA are available [5], and triangulation when the measurements of the AOA are available [6]. More generally, the mathematical solutions for indoor localization may be grouped into two categories: the probabilistic methods based on the Bayesian filtering process [7], [8], and the deterministic methods based on the optimization of a cost function. The work presented in this paper will focus on the latter due to their efficiency in terms of numerical complexity. These methods exploit the distances between the anchor nodes and the mobile in order to localize this one through solving an optimization problem. We include the absolute mobile moving direction provided by the LPS system, as an additional information about the mobile location. Therefore, these methods may be reformulated as a constrained optimization problem, and this constraint (mobile moving direction) will allow to narrow the search area of the mobile location in order to increase the accuracy, particularly for the one based on the Newton-Gauss algorithm [9], [10].

The rest of this paper is organized as follows. In Section II, the relative works of UWB LPS technologies and mathematical solutions are both discussed, with the side information on mobile direction. The Section III presents the proposed approach while in Section IV and V performance analysis and conclusion will be addressed, respectively.

\section{RELATED WORK}

\section{A. UWB-based local positioning system}

Since 2014 Microsoft has organized an indoor localization challenge at the IPSN conference [11]. For 3-D localization, UWB is the most used technology before sound and optical based solutions. A light weight (mobiles and anchors) and easy to set-up LPS system was recently developed for human tracking in [12]. Distances measurements between nodes are based on UWB technology using TOF with DecaWave DW1000 chip. Moreover, absolute orientation is given by a 9axis InvenSense inertial module unit coupled with embedded data fusion algorithm. Thanks to this information we can derive the mobile direction from its previous position, which is very helpful to enhance the accuracy of positioning algorithms as will be shown in Section III.

\section{B. Existing algorithms for indoor localization}

At each instant $t_{n}=n T$, with $T$ the sampling time, the mobile receives a set of signals from the $P$ anchors $\left\{A_{1}, \ldots, A_{P}\right\}$ located at known positions in the environment. These signals allow the measurements of a set of distances $\left\{d_{1, n}, \ldots, d_{P, n}\right\}$ that is linked to the unknown position $M_{n}$ (2-D position) through the following relationship

$$
d_{i, n}=\operatorname{dist}\left(A_{i}, M_{n}\right)+\eta_{d_{i, n}},
$$


where $\operatorname{dist}(X, Y)$ represents the distance between $X$ and $Y$, $\eta_{d_{i, n}}$ the measurement noise that can be supposed Gaussian. Localization algorithms can be grouped into the two following main families.

1) Bayesian methods: Recently, the Bayesian filtering process became a popular tool for indoor positioning [7]. The problem of interest in these methods is how estimating the current position of the mobile using both current and previous measurements. In other words, the estimation of the current position is achieved by considering the entire trajectory. For this purpose, a state equation that models the evolution in time of the mobile position as a discrete-time stochastic function is required and can be generally expressed as

$$
M_{n}=f_{n-1}\left(M_{n-1}, u_{n}\right),
$$

where $f_{n-1}($.$) is a known function and u_{n}$ represents the noise due to any mismodeling of the state equation. In the indoor localization context, a linear constant acceleration model has been generally used [13]. In Bayesian based methods, the information about the position of the mobile at time $n$ is modelled as the conditional probability distribution $p\left(M_{n} \mid d_{1}, \ldots, d_{n}\right)$, where $\mathbf{d}_{l}=\left\{d_{1, l}, \ldots, d_{P, l}\right\}$ is the set of measurements at time $1 \leq l \leq n$. Once this probability distribution is available, the position of the mobile can be considered as the Minimum Mean Square Error (MMSE) estimator

$$
\hat{M}_{n}=\mathbb{E}\left[\phi\left(M_{n}\right) \mid d_{1}, \ldots, d_{n}\right],
$$

for any measurable function $\phi($.$) . The filtering equation in-$ volved in Bayesian estimation can be analytically solved only in few cases, e.g. in case of linear Gaussian dynamic model systems where the Kalman filtering is an optimal solution. In other cases, a sub-optimal solution thanks to particle filtering or other alternatives of the Kalman filtering [7], [13], [14] is used. Although these solutions provide an interesting result in terms of accuracy, they require high numerical complexity, and then are not suitable for LPS with low energy resources.

2) Deterministic methods: These methods are based on the optimization of a cost function. The latter may be defined in order to solve a geometric problem [4], or from the relationship between the provided measurements, the anchors and the unknown mobile coordinates [10].

The trilateration is one of the first methods in mobile positioning problem. This method makes use of the point or surface of intersection formed by three circles of anchor nodes to determine the exact position, that leads to solve the following system of equations

$$
\left\{\begin{array}{l}
\left(x_{n}-x_{A_{i}}\right)^{2}+\left(y_{n}-y_{A_{i}}\right)^{2}=d_{i, n} \\
\left(x_{n}-x_{A_{j}}\right)^{2}+\left(y_{n}-y_{A_{j}}\right)^{2}=d_{j, n} \\
\left(x_{n}-x_{A_{k}}\right)^{2}+\left(y_{n}-y_{A_{k}}\right)^{2}=d_{k, n}
\end{array},\right.
$$

where $\left(\begin{array}{l}x_{A_{i}} \\ y_{A_{i}}\end{array}\right)$ and $\left(\begin{array}{l}x_{n} \\ y_{n}\end{array}\right)$ means the 2-D coordinates of the anchor node $A_{i}$ and the unknown position of $M_{n}$, respectively. The anchor nodes $A_{i}, A_{j}$ and $A_{k}$ are supposed to be not aligned. Several strategies are proposed in order to efficiently solve (4). In [3], [15] the authors formulate the trilateration as a least square problem, while others strategies consist in finding the best anchor nodes [16]. Although these methods are very promising for low-energy resource applications, they remain very sensitive to the noise affecting the provided measurements $\mathbf{d}_{n}$. Note that when many anchors are available, it is possible to perform trilateration on all non-aligned anchor triplets. However, this method called as multilateration is very complex, thus, it is not suitable for LPS with low complexity algorithms.

To take advantage of all available data in order to improve the performance without increasing the complexity, one method seems to stand out among all ones based on minimization of a cost function. The latter, is based on the Newton-Gauss algorithm for non linear function [9], and as in [10], consists in finding the minimum of the following cost function

$$
F\left(x_{n}, y_{n}\right)=\sum_{i=1}^{P}\left(\sqrt{\left(x_{n}-x_{A_{i}}\right)^{2}+\left(y_{n}-y_{A_{i}}\right)^{2}}-d_{i, n}\right)^{2} .
$$

In [10], in order to improve the localization accuracy, a modified Newton-Gauss method has been developed. Compared to the classical method [9], the authors developed a geometric intersection model to narrow the targeted search area, and also allow a self-correction of bad measurements data. This method outperforms the classical one in terms of localization accuracy, but leads to a significant increase of numerical complexity. As in [10], the solution presented in this article proposes to narrow the targeted search area in the Newton-Gauss method. For this purpose, the estimation of the mobile moving direction provided by the LPS will be used as a constraint in the considered optimization problem. This direction allows to narrow the targeted area directly without additional computing steps. Therefore, this novel strategy will allow to increase the localization accuracy without increasing significantly the numerical complexity.

\section{PROPOSED SOLUTION}

At each instant $t_{n}=n T$, let $\mathbf{e}_{n}$ be the estimation of the unit moving vector $\frac{\overrightarrow{M_{n-1} M_{n}}}{\| \overrightarrow{M_{n-1} M_{n} \|}}$ of the mobile from its previous position $M_{n-1}$. In this paper, we propose to use both the set of measurements $\mathbf{d}_{n}$ and $\mathbf{e}_{n}$ in order to estimate the position of the mobile unlike classical deterministic methods, wherein only the set $\mathbf{d}_{n}$ is exploited. The choice of the method based on the Newton-Gauss algorithm [9] with the cost function defined by (5) is motivated by the fact that this method allows a better deal between localization accuracy and numerical complexity among the deterministic methods. As it is briefly mentioned in Section II, this method, as the one presented in [10], consists in finding the minimum of (5), i.e.

$$
\hat{M}_{n}=\arg \min _{M}\left(\sum_{i=1}^{P}\left(\operatorname{dist}\left(A_{i}, M\right)-d_{i, n}\right)^{2}\right) .
$$

It is important to highlight that when the measured distances $\mathbf{d}_{n}$ are misestimated, the performance of this method decreases significantly. The reasons for this behavior can be various:

1) the cost function is not a quadratic linear form, therefore the convergence toward the optimal solution is not 
guaranteed and can highly depend on the initial point used by Newton-Gauss descent algorithm [9].

2) many local optimum solutions can exist and the stopping criterion did not allow us to choose one of them.

A simple strategy to overcome these drawbacks may consist in including a constraint in an optimization problem to narrow the useful search area. For this purpose, thanks to the additional information about the location of $M_{n}$, a DirectionAided Newton-Gauss (DA-NG) based method is proposed. This method proposes to minimize the same cost function subject to the unit moving direction $\mathbf{e}_{n}$ of the mobile, that leads to the following optimization problem

$$
\hat{M}_{n}=\left\{\begin{array}{c}
\arg \min _{M}\left(\sum_{i=1}^{P}\left(\operatorname{dist}\left(A_{i}, M\right)-d_{i, n}\right)^{2}\right) \\
\text { s.t. } \overrightarrow{\hat{M}_{n-1} M} \propto \mathbf{e}_{n}
\end{array} .\right.
$$

The interest of the constraint is to allow a better accuracy of the Newton-Gauss algorithm by compelling the algorithm to seek the optimal solution in a restricted domain. However, when the data $\mathbf{d}_{n}$ are more reliable than the provided direction measurement, the algorithm will produce the opposite effect, i.e. a worse accuracy. In practice, we have the estimated position $\hat{M}_{n-1}$ and not the true position $M_{n}$, which implies that the direction considered may be more noisy than the one provided by the system. Therefore, to prevent the inconvenience that may arise from this fact, we propose to "smooth" the constraint in the optimization problem (7). For this purpose, let $\mathcal{S}_{\left[\omega, \mathbf{e}_{n}\right]}$ be the angular surface defined as follow,

$$
\mathcal{S}_{\left[\omega, \mathbf{e}_{n}\right]}=\left\{M, \frac{\overrightarrow{\| \hat{M}_{n-1} M}-\left\langle\overrightarrow{\hat{M}_{n-1} M}, \mathbf{e}_{n}\right\rangle \mathbf{e}_{n} \|}{\left\|\left\langle\overrightarrow{\hat{M}_{n-1} M}, \mathbf{e}_{n}\right\rangle \mathbf{e}_{n}\right\|} \leq|\tan (\omega)|\right\}
$$

where $\omega \geq 0$ is fixed; for instance $\omega$, which denotes the maximum tolerated angular derivation w.r.t. the vector $\mathbf{e}_{n}$, may be chosen in $[0, \pi / 4]$. In Fig. 1, an illustration of the influence of the choice of $\omega$ w.r.t. $\mathcal{S}_{\left[\omega, \mathbf{e}_{n}\right]}$ is shown. Intuitively, if $\hat{M}_{n-1}$ is accurately estimated, then it can be easily noticed that the best $\omega$ is equal to $\left(\widehat{\mathbf{e}_{n}^{*}, \mathbf{e}_{n}}\right)$ with $\mathbf{e}_{n}^{*}$ the true direction and $\mathbf{e}_{n}$ the estimated one. Indeed, this choice leads to use the smallest area containing the real mobile moving direction, i.e. $\mathbf{e}_{n}^{*}$.

So, the proposed DA-NG method consists in minimizing

$$
\hat{M}_{n}=\arg \min _{M \in \mathcal{S}_{\left[C, \mathbf{e}_{n}\right]}}\left(\sum_{i=1}^{P}\left(\operatorname{dist}\left(\mathcal{A}_{i}, M\right)-d_{i, n}\right)^{2}\right) .
$$

It can be noticed that (7) is equivalent to (9) when $\omega=0$. The numerical solution of (9) is described in Algorithm 1.

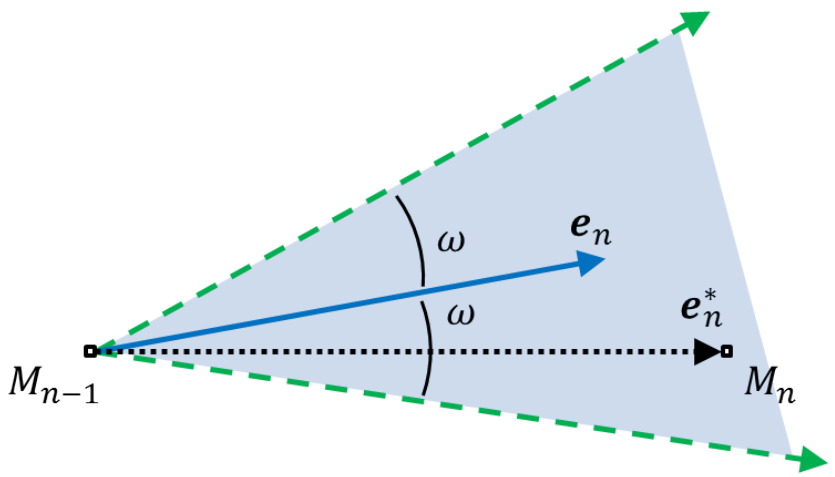

Fig. 1. Illustration of the narrowed useful search area.

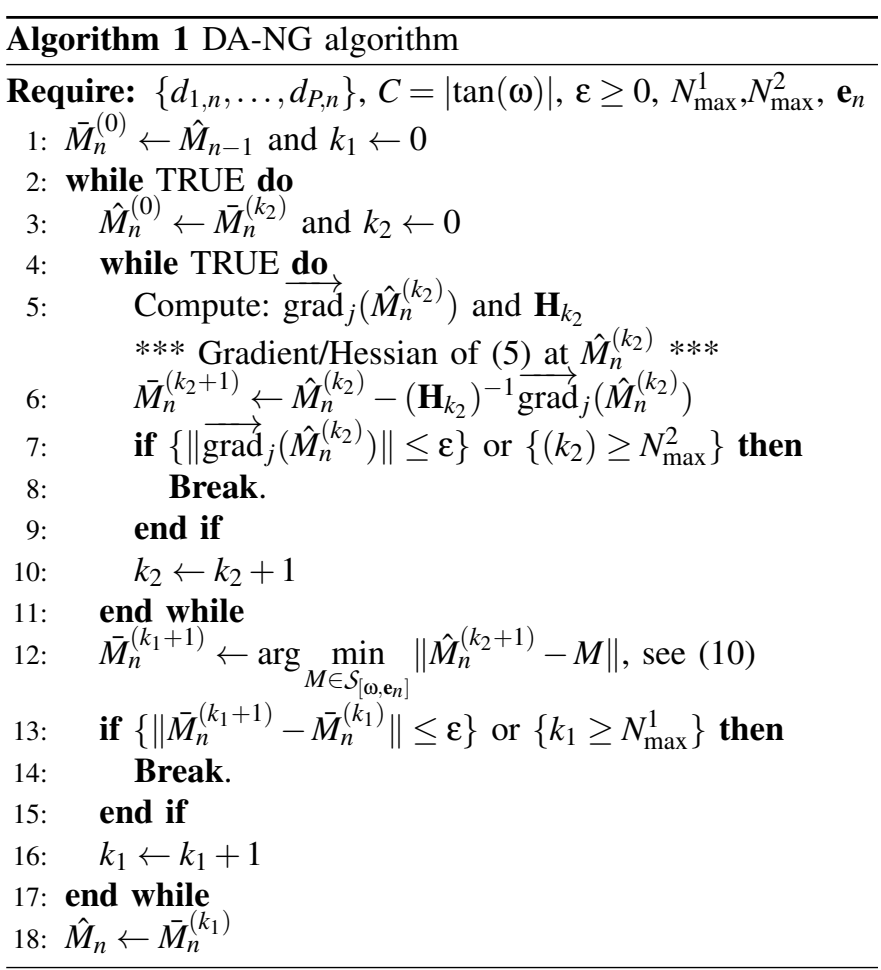

Let $\alpha$ and $\beta$ be the scalar product $\left\langle\overrightarrow{\hat{M}_{n}^{\left(k_{2}+1\right)} \hat{M}_{n-1}}, \mathbf{e}_{n}\right\rangle$ and $\left\langle\overrightarrow{\hat{M}_{n}^{\left(k_{2}+1\right)} \hat{M}_{n-1}}, \mathbf{e}_{n}^{\perp}\right\rangle$, respectively. Therefore, after some derivation we have

$$
\bar{M}_{n}^{\left(k_{1}+1\right)}=\bar{\alpha} \mathbf{e}_{n}+\bar{\beta} \mathbf{e}_{n}^{\perp}+\hat{M}_{n-1},
$$

where $(\bar{\alpha}, \bar{\beta})$ is given as follows

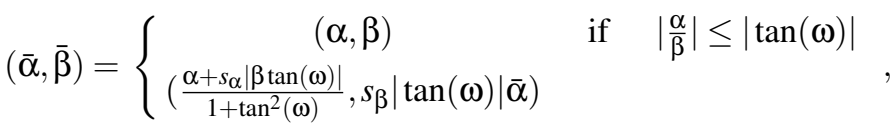

where $s_{x}$ means the sign of $x$. From Algorithm 1 it can be noticed that the constraint is applied once the classical Newton-Gauss method converges i.e. satisfies the stopping criteria. This choice allows to entirely take advantage of 
the classical Newton-Gauss method [9], [10]. Indeed, when the constraint is applied after each iteration of the classical method, i.e. when $N_{\max }^{2}=1$, the proposed method may be unstable and therefore becomes less robust than the one proposed in [10]. The second important point to highlight is the influence of the constraint choice $\omega$. To do this, let consider the simulation model (serpentine in a square room of size $15 \times 25$ ) depicted in Fig. 2 where the green line denotes the real trajectory and the circle (blue) the position of the anchors in the room. To illustrate the movement of the mobile along the trajectory, we will randomly sample the latter such that $\left\|M_{n}-M_{n-1}\right\| \sim \mathcal{U}\left(\left[d_{\min }, d_{\max }\right]\right)$ where $d_{\min }=0.2 \mathrm{~m}$ and $d_{\max }=0.5 \mathrm{~m}$. We assume that $d_{i, n}=d_{i, n}^{*}+\eta_{d_{i, n}}$ where $d_{i, n}^{*}=\operatorname{dist}\left(A_{i}, M_{n}\right)$ is the real distance and $\eta_{d_{i, n}} \sim \mathcal{N}\left(0, \sigma_{d}\right)$, and $\mathbf{e}_{n}=\left(\begin{array}{c}\cos \left(\theta_{n}^{*}+\eta_{\theta_{n}}\right) \\ \sin \left(\theta_{n}^{*}+\eta_{\theta_{n}}\right)\end{array}\right)$ where $\eta_{\theta_{n}} \sim \mathcal{N}\left(0, \sigma_{\theta}\right)$ and $\mathbf{e}_{n}^{*}=\left(\begin{array}{c}\cos \left(\theta_{n}^{*}\right) \\ \sin \left(\theta_{n}^{*}\right)\end{array}\right)$ means the real direction. Note that $\sigma_{d}$ and $\sigma_{\theta}$ represent standard deviation of the noise on distances and mobile moving direction, respectively.

Fig. 3 and Fig. 4 compare the DA-NG versus $\omega$ with the one proposed in [10] in terms of localization accuracy

$$
\Delta=\arg \min _{\delta>=0}\left\{\operatorname{Prob}[\varepsilon \geq \delta] \leq 10^{-3}\right\},
$$

where $\varepsilon$ means the relative error square between the real position of the mobile and the one after localization.

As it is expected, the simulation results depicted in Fig. 3 and Fig. 4 show that the choice of the constraint is both crucial and delicate. It can be noticed that:

- each $\omega$ allows to improve the performance of the classical Newton-Gauss method except when $\tan (\omega)$ is close to zero or high. That is trivial because in these cases, the constraint becomes inactive.

- the performance of the DA-NG method in terms of localization accuracy versus $\omega$ is not monotone, which means that the choice of the best constraint $\omega$ is not intuitive.

- the more the noise is affecting the measurements, the higher gain over classical methods is achieved by DANG.

It is also important to note that the performance of the DANG method does not depend significantly on $\sigma_{\theta}$. That is pre-

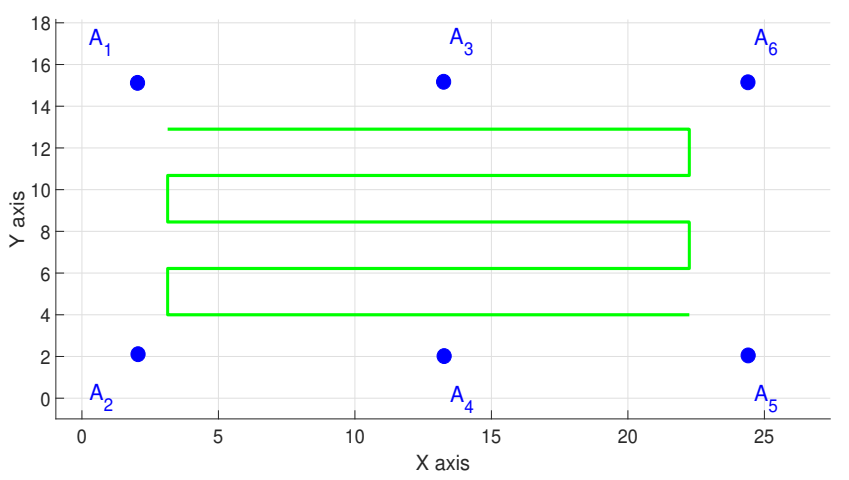

Fig. 2. Simulation model.

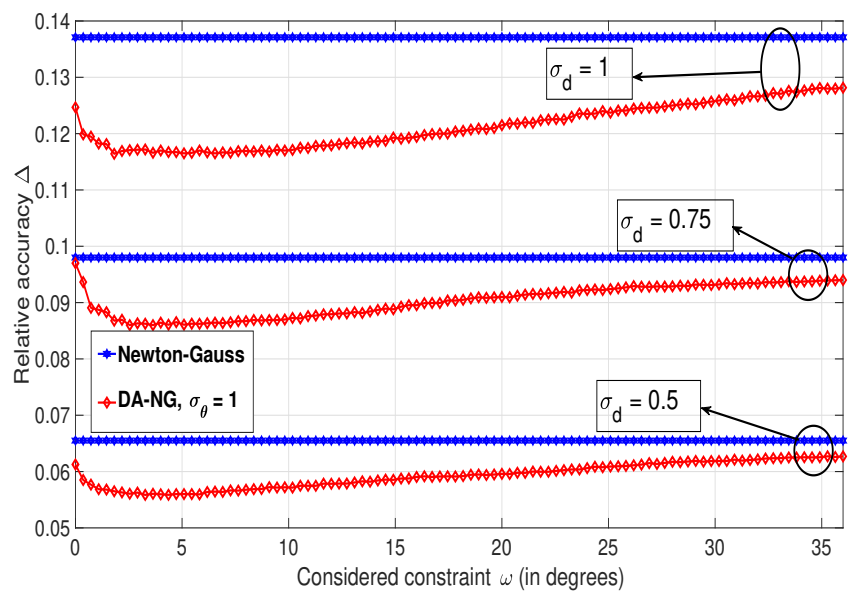

Fig. 3. Localization accuracy w.r.t. $\omega$ when $\sigma_{\theta}=1$ degree.

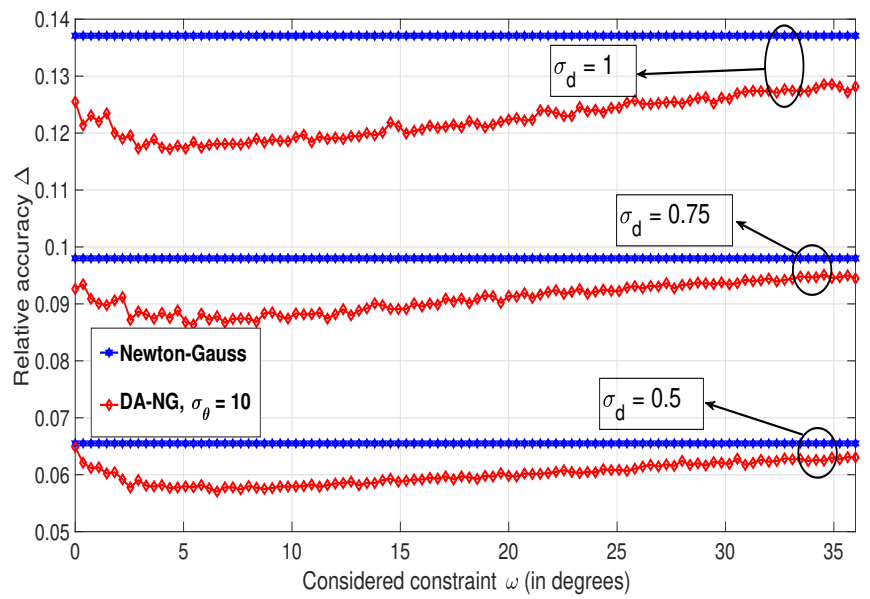

Fig. 4. Localization accuracy w.r.t. $\omega$ when $\sigma_{\theta}=10$ degrees.

dictable, since in practice the point $M_{n-1}$ is not known, thus, an additional noise is added to the one from the measurement of the direction $\mathbf{e}_{n}$.

\section{Performance analysis}

In this section, both DA-NG and classical methods are compared in terms of numerical complexity and localization accuracy. For the latter, the relative accuracy defined by (12) is used as performance criterion. The choice of relative accuracy allows to ignore the considered domain in our simulation results.

\section{A. Numerical complexity analysis}

Without loss of generality, the numerical complexity will be evaluated in terms of number of float additions (ad), multiplications or divisions $(m d)$ and square roots $(s r)$. Let $\mathcal{C}_{1}$ be the numerical complexity of the Newton-Gauss method after each iteration. Thus, from Algorithm 1 we have

$$
\mathcal{C}_{1}=11(P+1) m d+(12 P+5) a d+P s r .
$$




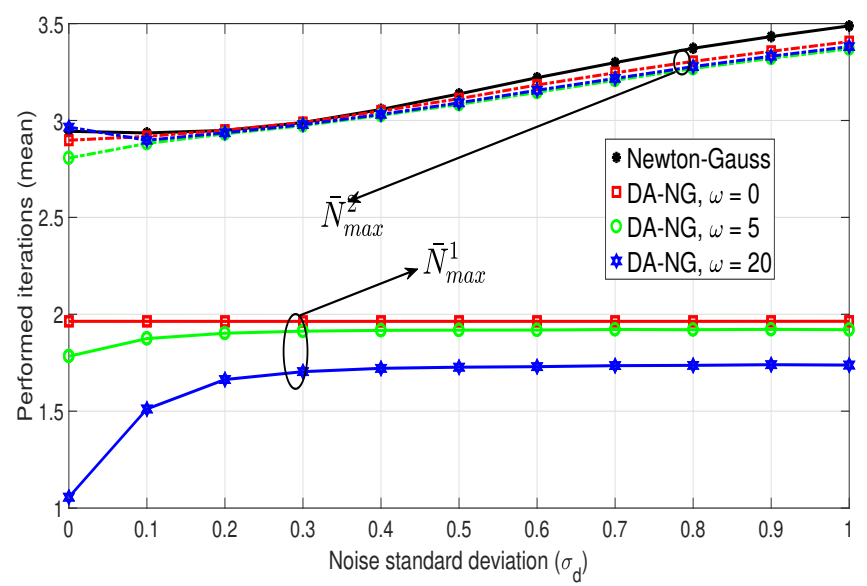

Fig. 5. Iterations mean in Newton-Gauss method $\left(\bar{N}_{\max }\right)$ and DA-NG method $\left(\bar{N}_{\max }^{1}, \bar{N}_{\max }^{2}\right)$ versus $\sigma_{d}$.

Therefore, the numerical complexity of the classical NewtonGauss method and the DA-NG method are given by (14) and (15), respectively.

$$
\begin{aligned}
\mathcal{C}_{N G} & =\bar{N}_{\max } \mathcal{C}_{1}, \\
\mathcal{C}_{D A-N G} & =\bar{N}_{\max }^{1}(\bar{N}_{\max }^{2} \mathcal{C}_{1}+\underbrace{(8 a d+13 m d)}_{\mathcal{C}_{2}}),
\end{aligned}
$$

where $\bar{N}_{\max }$ is the mean of iterations performed by the classical Newton-Gauss method, $\bar{N}_{\max }^{1}$ and $\bar{N}_{\max }^{2}$ represent the mean of iterations performed by the DA-NG method in the first and second loop, respectively. The second terms in (15) represents the numerical complexity required to apply the constraint after each iteration in the first loop, see (11). For all presented simulation results in this section and in next ones, we have $N_{\max }=N_{\max }^{2}=20$ and $N_{\max }^{1}=5$. The choice of a high $N_{\max }$ allows to guarantee that each solution of the classical NewtonGauss method (or the output point after the second loop in Algorithm 1 satisfies the necessary condition for optimality. However, in practice, a low number of iterations is generally required as it is depicted in Fig. 5, wherein $\mathbb{E}\left[N_{\max }\right]=\bar{N}_{\max }$, $\mathbb{E}\left[N_{\max }^{1}\right]=\bar{N}_{\max }^{1}$ and $\mathbb{E}\left[N_{\max }^{2}\right]=\bar{N}_{\max }^{2}$ are computed over $10^{5}$ trials.

From the simulation results depicted in Fig. 5, it can be noticed that the DA-NG method and the classical method have a very low numerical complexity. Indeed, from the results depicted in Fig. 5 and (15), we note that the proposed solution is at most twice more complex than the classical method, since $\mathcal{C}_{2} \ll \mathcal{C}_{1}$.

\section{B. Accuracy performance}

In this section, we analyze the performance of the DANG method compared to the classical method. In Fig. 6 and Fig. 7 we can see the influence of the quality of the provided measurements $\mathbf{d}_{n}$. The simulation model is the one used in Section III, see Fig. 2. For statistical reason, the accuracy has been computed with $10^{5}$ trials.

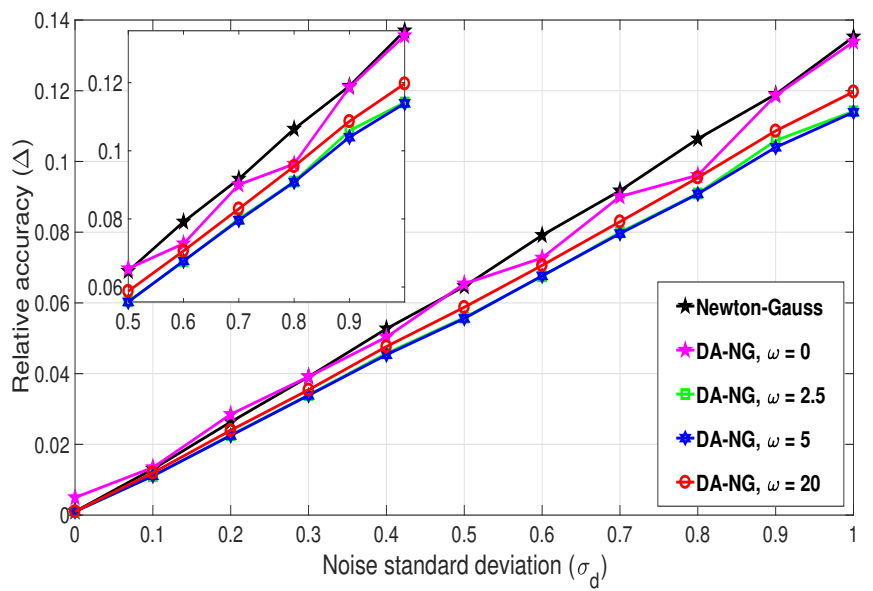

Fig. 6. Localization accuracy w.r.t. $\sigma_{d}$ when $\sigma_{\theta}=1$ degree.

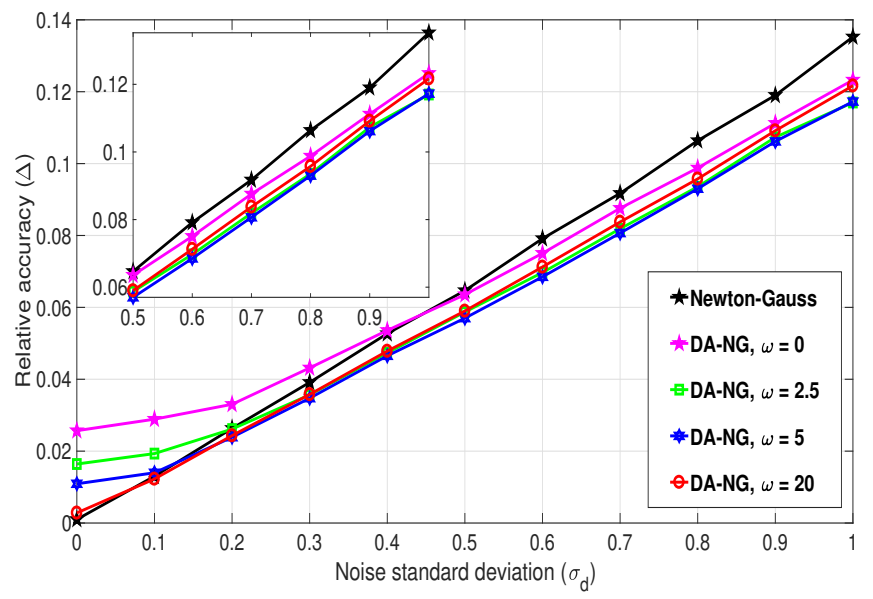

Fig. 7. Localization accuracy w.r.t. $\sigma_{d}$ when $\sigma_{\theta}=10$ degrees.

The results in Fig. 6 and Fig. 7 show that the DANG outperforms the classical method when the data $\mathbf{d}_{n}$ are misestimated. However, when the measurements data are accurately estimated, the DA-NG becomes less accurate than the classical, and more especially when the provided direction is misestimated, as illustrated by Fig. 7. As it is remarked in Section III, the simulation results confirm that the choice of the constraint $\omega$ to be applied is very important, and can allow a high improvement of the accuracy. Indeed, we remark when the used constraint $\omega$ is close to zero or very large, the accuracy of the DA-NG method decreases. Indeed, in the case of the provided measurements are misestimated (both anchors-Mobile distances and moving direction), for instance $0.5 \leq \sigma_{d} \leq 1$ and $\sigma_{\theta}=10$ degrees, the proposed method allows to achieve a gain (on average) in term of accuracy over classical method of about $6.1 \%, 12.7 \%, 14.1 \%$ and $10.3 \%$ when the applied constraint is $\omega=0,2.5,5$ and 20 degrees, respectively. Thus, it can be concluded that the DA-NG method allows a gain of more than $14 \%$ when an adequate constraint is applied. Furthermore, we remark that a good choice of the constraint allows to reduce the influence of a misestimation 


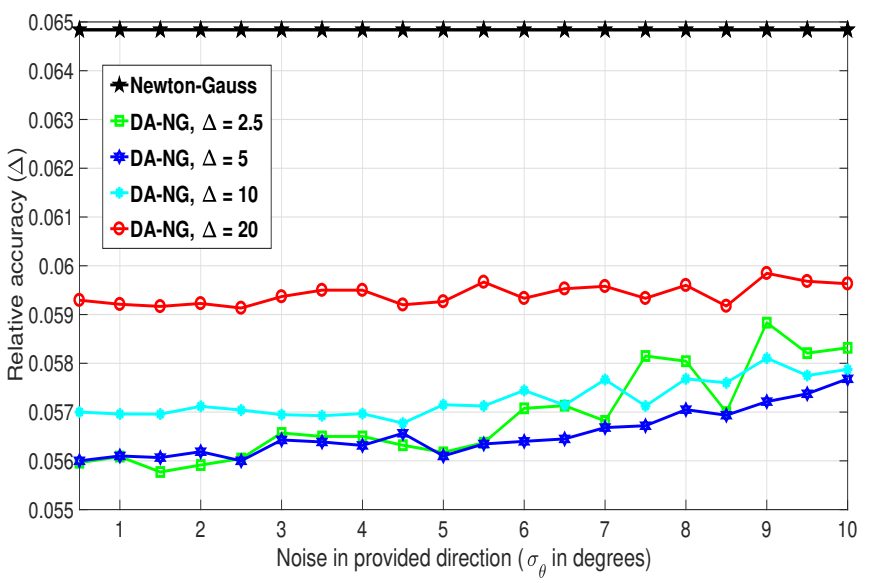

Fig. 8. Influence of $\sigma_{\theta}\left(\sigma_{d}=0.5\right)$.

of the direction, this can be confirmed by the simulation results depicted in Fig. 8, where the performance of the DANG method is evaluated versus the accuracy of the provided moving direction i.e. vs $\sigma_{\theta}$.

These results show that the DA-NG method is robust against the noise affecting the measured direction. In fact, we remark from Fig. 8 that the performance of the proposed DA-NG method decreases slowly when $\omega$ increases, and they remain better than the one of the classical method.

\section{CONCLUSION}

We have proposed in this paper a direction-aided NewtonGauss method for indoor positioning. This method proposes to use the mobile moving direction to narrow the search area in order to increase the accuracy of the classical Newton-Gauss method. The simulation results presented in this paper showed that this strategy allows to achieve a gain higher than $14 \%$ in accuracy over the classical method, and with only a slight increase of the numerical complexity. As a further work, the seeking area can be adapted at each point to increase accuracy and performance efficiency. In this case, this area will depend on the difference between successive measured directions.

\section{REFERENCES}

[1] R. F. Brena, J. P. García-Vázquez, C. E. Galván-Tejada, D. MuñozRodriguez, C. Vargas-Rosales, and J. Fangmeyer Jr, "Evolution of indoor positioning technologies: A survey," Journal of Sensors, 2017.

[2] Z. Farid, R. Nordin, and M. Ismail, "Recent advances in wireless indoor localization techniques and system," Journal of Computer Networks and Communications, 2013.

[3] F. Seco, A. R. Jiménez, C. Prieto, J. Roa, and K. Koutsou, "A survey of mathematical methods for indoor localization," in IEEE International Symposium on Intelligent Signal Processing (WISP), Aug. 2009, pp. 9 14.

[4] M. E. Rusli, M. Ali, N. Jamil, and M. M. Din, "An improved indoor positioning algorithm based on rssi-trilateration technique for internet of things (iot)," in IEEE International Conference on Computer and Communication Engineering (ICCCE), July 2016, pp. 72-77.

[5] D. Ruiz, J. Ureña, J. C. García, C. Pérez, J. M. V. Carrizo, and E. García, "Efficient trilateration algorithm using time differences of arrival," Sensors and Actuators A: Physical, vol. 193, pp. 220-232, 2013.

[6] P. Rong and M. L. Sichitiu, "Angle of arrival localization for wireless sensor networks," in IEEE Conference on Sensor and Ad Нос Соттиnications and Networks (SECON), Sept. 2006, pp. 374-382.

[7] F. Gustafsson, "Particle filter theory and practice with positioning applications," IEEE Aerospace and Electronic Systems Magazine, vol. 25 , no. 7, pp. 53-82, 2010.

[8] G.-M. Hoang, M. Gautier, and A. Courtay, "Cooperative-cumconstrained maximum likelihood algorithm for uwb-based localization in wireless bans," in IEEE International Conference on Communications (ICC), June 2015

[9] J. Nocedal and S. J. Wright, "Numerical optimization 2nd," 2006.

[10] S. Huang, Z. Wu, and A. Misra, "A practical, robust and fast method for location localization in range-based systems," Sensors, vol. 17, no. 12, p. 2869, 2017.

[11] "Microsoft indoor localization competition - ipsn 2018." [Online]. Available: https://www.microsoft.com/en-us/research/event/ microsoft-indoor-localization-competition-ipsn-2018/

[12] A. Courtay, M. Le Gentil, O. Berder, A. Carer, P. Scalart, and O. Sentieys, "Zyggie: A wireless body area network platform for indoor positioning and motion tracking," in IEEE International Symposium on Circuits and Systems (ISCAS), May 2018.

[13] A. Dhital, P. Closas, and C. Fernández-Prades, "Bayesian filtering for indoor localization and tracking in wireless sensor networks," EURASIP Journal on Wireless Communications and Networking, vol. 2012, no. 1, p. 21, 2012.

[14] J. Míguez, M. F. Bugallo, and P. M. Djurić, "A new class of particle filters for random dynamic systems with unknown statistics," EURASIP Journal on Applied Signal Processing, vol. 2004, pp. 2278-2294, 2004.

[15] Y. Zhou, "An efficient least-squares trilateration algorithm for mobile robot localization," in IEEE International Conference on Intelligent Robots and Systems (IROS), Oct. 2009, pp. 3474-3479.

[16] O. Artemenko, T. Simon, A. Mitschele-Thiel, D. Schulz, and R. S. Ta, "Comparison of anchor selection algorithms for improvement of position estimation during the wi-fi localization process in disaster scenario," in IEEE Conference on Local Computer Networks (LCN), Oct. 2012, pp. $44-49$. 